\title{
INTERAÇÕES MEDICAMENTOSAS POTENCIAIS NA FARMACOTERAPIA DE IDOSOS ATENDIDOS EM FARMÁCIA BÁSICA DO SUL DO BRASIL
}

\author{
POTENTIAL DRUG INTERACTIONS IN THE \\ PHARMACOTHERAPY OF ELDERLY ATTENDED \\ IN BASIC PHARMACY IN SOUTHERN BRAZIL
}

\section{Cristiane Carla Dressler Garske', Mariana Portela de Assis², Ana Paula Helfer Schneider², Ediberto de Oliveira Machado4, Lisoni Muller Morsch ${ }^{5}$}

\section{RESUMO}

Interação medicamentosa indesejável está entre as principais causas dos problemas relacionados aos medicamentos, podendo ocasionar prejuízos na recuperação da saúde do paciente. 0 objetivo foi investigar a ocorrência de interações medicamentosas na farmacoterapia dos idosos atendidos na Farmácia Básica de Santa Cruz do Sul - RS. Estudo transversal, com coleta de dados entre Agosto a Setembro de 2014, através de entrevista individual. As interações medicamentosas foram obtidas através do software Micromedex ${ }^{\circledR}$ Solutions. Dos 204 regimes terapêuticos analisados, $54,4 \%$ continham pelo menos uma interação medicamentosa. A gravidade das interações encontradas foi: menor $(n=26)$, moderada $(n=160)$ e maior $(n=45)$. O Ácido Acetilsalicílico teve a maior prevalência $(23,8 \%)$ de interações, e Hidroclorotiazida foi o mais prescrito (18,6\%). O manejo clínico mais indicado para as interações foi monitorização do paciente $(73,6 \%)$. A identificação das interações permite tratamentos mais efetivos, menos complicações, racionalidade dos medicamentos, melhorias na qualidade das prescrições e na qualidade de vida dos pacientes.

Descritores: Idosos; Farmacoterapia; Interações Medicamentosas.

\begin{abstract}
The undesirable drug interactions is among the main causes of the problems related to drugs and may cause losses in the recovery of the patient's health. The objective was to investigate the occurrence of drug interactions in the pharmacotherapy of the elderly attended in Basic Pharmacy in Santa Cruz do Sul - RS. Cross-sectional study with data collection from August to September 2014, through individual interviews. Drug interactions were obtained by Micromedex ${ }^{\circledR}$ Solutions software. $54.4 \%$ of the 204 therapeutic regimens analyzed had at least one drug interaction. The severity of the interactions found was: minor $(n=26)$, moderate $(n=160)$ and major $(n=45)$. The Aspirin had the highest prevalence (23.8\%) of interactions and Hydrochlorothiazide was the most prescribed (18.6\%). The most appropriate clinical management of interactions was the patient's monitoring (73.6\%). The identification of the interactions allows treatments more effective, fewer complications, rationality of drugs, improvements in the quality of prescriptions and in the quality of patients life.
\end{abstract}

${ }^{1}$ Graduada em Farmácia pela Universidade de Santa Cruz do Sul (UNISC), Santa Cruz do Sul, RS, Brasil.

${ }^{2}$ Graduada em Farmácia pelo Centro Universitário Univates (UNIVATES), Lajeado, RS, Brasil.

${ }^{3}$ Mestre em PPG Saúde Coletiva pela Universidade do Vale do Rio dos Sinos (UNISINOS),

São Leopoldo, RS, Brasil.

${ }^{4}$ Mestre em Desenvolvimento Regional pela Universidade de Santa Cruz do Sul (UNISC), Santa Cruz do Sul, RS, Brasil.

${ }^{5}$ Mestre em Ciência e Tecnologia Farmacêuticas pela Universidade Federal de Santa Maria (UFSM), Santa Maria, RS, Brasil. 


\section{Introdução}

AOrganização Mundial de Saúde(OMS) defende que a população idosa crescerá de tal forma, que o Brasil futuramente ocupará o sexto país do mundo com maior número de idosos, podendo alcançar cerca de 15 milhões de pessoas com 60 anos ou mais no ano de 20201; passando de 7,4\% do total em 2013 para 26,7\% do total em 2060². Estima-se que $70 \%$ das pessoas idosas fazem uso de medicamentos e cerca de $20 \%$ destas consomem em média três medicamentos concomitantes. A polifarmácia, definida como o uso de cinco ou mais medicamentos ${ }^{3}$, pode propiciar o aumento do uso de medicamentos inadequados, induzindo à subutilização de medicamentos essenciais para o adequado controle de condições prevalentes nos idosos, a possibilidade de erros de administração como os esquecimentos dos horários de administração dos medicamentos prescritos ${ }^{4}$. A prevalência de doenças crônicas não transmissíveis que requerem a associação de vários medicamentos e a forma como é realizada a assistência à saúde do idoso, por diferentes especialistas que desconhecem o seu histórico medicamentoso, explica em muitas situações a polifarmácia ${ }^{5}$. 0 idoso encontra desta forma uma barreira para a adesão aos seus tratamentos, na medida em que tornam complexos os esquemas terapêuticos, e a possibilidade da ocorrência de interações medicamentosas e reações adversas ${ }^{6}$.

A interação medicamentosa é a resposta farmacológica ou clínica oriunda da interferência da ação de um determinado medicamento, alimento ou qualquer substância química sobre o efeito de outro medicamento, administrado previamente ou em concomitância ao primeiro ${ }^{7,8}$. As alterações fisiológicas que ocorrem com 0 avançar da idade também contribuem para que estas aconteçam em maior proporção, tais alterações referem-se à produção de suco gástrico diminuída, esvaziamento gástrico mais lento, teor de água total menor, teor de tecido adiposo total maior, menor quantidade de proteínas plasmáticas, diminuição da irrigação renal, filtração glomerular e secreção tubular ${ }^{9}$, redução do fluxo sanguíneo e das atividades enzimáticas no fígado ${ }^{10}$, entre outras, que podem induzir à manifestação de interações farmacocinéticas, possibilitando a ocorrência de interações medicamentosas que podem aumentar os efeitos indesejados dos medicamentos, acarretar em ineficácia terapêutica e/ou repercutir negativamente na saúde do idoso, acarretando em custos elevados com o tratamento e até mesmo evoluir para o óbito do indivíduo ${ }^{11}$.

A ocorrência de interações medicamentosas é um dos principais riscos envolvidos no uso de medicamentos por idosos $^{12}$. Muitas vezes elas não são identificadas, pois são mascaradas pelas alterações fisiológicas, comorbidades e 0 grande número de medicamentos usados pelos idosos, sendo algumas vezes confundidas com efeitos adversos próprios de um dos medicamentos utilizados, um agravamento do estado de saúde, um curso normal das comorbidades ou a ineficácia do tratamento ${ }^{13}$. Essa situação evidencia um importante problema de saúde pública que deve ser cuidadosamente acompanhado ${ }^{14}$, já que os idosos são mais suscetíveis aos efeitos adversos e às interações medicamentosas ${ }^{15}$. Diante do exposto, o objetivo deste estudo foi investigar a ocorrência das interações medicamentosas potenciais na farmacoterapia utilizada pelos idosos atendidos na Farmácia Básica de Santa Cruz do Sul - RS, em termos de sua prevalência e das características a elas associadas.

\section{Metodologia}

Estudo de delineamento observacional e transversal. A população alvo foi constituída de 300 idosos escolhidos aleatoriamente, de ambos os sexos, atendidos na Farmácia Básica de Santa Cruz do Sul - RS, no período de Agosto e Setembro de 2014. Cabe destacar que apenas os regimes terapêuticos foram avaliados não havendo acompanhamento dos idosos os quais foram convidados a participar do estudo no momento da dispensação. Após concordância em participar da pesquisa foi realizada a leitura e assinado o termo de consentimento livre e esclarecido. A pesquisa foi conduzida de acordo com os padrões éticos exigidos e aprovada pelo Comitê de Ética em Pesquisa da Universidade de Santa Cruz do Sul no dia 26/08/14 sob o número 764.946.

Os dados foram coletados mediante entrevista individual com questionário semiestruturado, validado em estudo piloto, contendo dados da farmacoterapia. As possíveis interações medicamentosas entre medicamentos foram verificadas através da base de dados Micromedex ${ }^{\circledR}$ Solutions $(2012)^{16}$.

Foram excluídos do estudo ( $n=96$ ) os regimes terapêuticos que continham menos de dois medicamentos e/ou apresentavam medicamentos não encontrados na base de dados. Foram contabilizadas como interações medicamentosas potenciais, as que apresentavam documentação excelente ou boa quanto ao conhecimento cientifico existente a seu 
respeito e aquelas classificadas segundo o impacto da interação sobre o paciente em níveis de gravidade contraindicado, importante (maior), moderada e secundária (menor), sendo considerado sem interação medicamentosa quando a documentação era classificada como razoável ou desconhecida. Posteriormente os dados foram digitados no Microsoft Exce $^{\circledR} 2013$ e analisados no software SPSS versão 20.0 ano 2011 (Statistical Package for the Social Sciences 20.0).

\section{Resultados}

Nos 204 regimes terapêuticos pesquisados foram encontrados 797 medicamentos, apresentando uma média de 3,9 medicamentos por idoso. O número de medicamentos utilizado pelos idosos variou de 2 a 11, sendo que 32,3\% faziam uso de cinco ou mais medicações, caracterizando como polifarmácia. Os medicamentos mais utilizados pelos idosos foram os relacionados aos problemas do sistema cardiovascular $(42,9 \%)$ seguido dos que atuavam no sistema nervoso central $(20,9 \%)$. Constatou-se que $54,4 \%$ das prescrições médicas ( $n=111)$ continham pelo menos uma interação medicamentosa potencial. Na Tabela 1 está descrita a relação entre o número de medicamentos prescritos e presença de interações medicamentosas.

Tabela 1 - Relação entre o número de medicamentos prescritos e presença de interações

\begin{tabular}{c|c|c|c}
\hline $\begin{array}{c}\text { Número de medicamentos } \\
\text { por prescrição médica }\end{array}$ & $\begin{array}{c}\text { Número de } \\
\text { idosos }\end{array}$ & $\begin{array}{c}\text { Número de } \\
\text { prescrições médicas } \\
\text { com interação }\end{array}$ & $\begin{array}{c}\% \text { de interações } \\
\text { medicamentosas }\end{array}$ \\
\hline Até 5 & 172 & 83 & 48,8 \\
\hline De 6 a 10 & 31 & 27 & 87,1 \\
\hline Mais de 10 & 1 & 1 & 100 \\
\hline Total & 204 & 111 & - \\
\hline
\end{tabular}

No Quadro 1 estão descritas as interações medicamentosas potenciais encontradas, prevalência e classificação de acordo com a sua gravidade e os efeitos que podem causar no paciente.

Quadro 1 - Interações medicamentosas potenciais de acordo com a sua gravidade

\begin{tabular}{|c|c|c|}
\hline Interação medicamentosa & Prevalência & Mecanismo/Efeito \\
\hline \multicolumn{3}{|c|}{ Interação Secundária (Menor) n=26 } \\
\hline $\begin{array}{l}\text { Alendronato de Sódio + } \\
\text { Carbonato de Cálcio }\end{array}$ & 12 & $\begin{array}{l}\text { Pode reduzir a absorção do Alendronato } \\
\text { de Sódio. }\end{array}$ \\
\hline Diazepam + Fluoxetina & 6 & $\begin{array}{c}\text { Pode resultar em maiores concentrações } \\
\text { séricas de Diazepam. }\end{array}$ \\
\hline Diazepam + Omeprazol & 5 & $\begin{array}{l}\text { Pode prolongar ou aumentar os efeitos } \\
\text { do Diazepam. }\end{array}$ \\
\hline $\begin{array}{c}\text { Atenolol + Carbonato de } \\
\text { Cálcio }\end{array}$ & 2 & Pode reduzir a eficácia do Atenolol. \\
\hline Amitriptilina + Fenobarbital & 1 & $\begin{array}{l}\text { Pode diminuir as concentrações séricas } \\
\text { de Amitriptilina. }\end{array}$ \\
\hline \multicolumn{3}{|c|}{ Interação Moderada $\quad n=160$} \\
\hline Captopril + Hidroclorotiazida & 15 & $\begin{array}{l}\text { Pode resultar em hipotensão postural } \\
\text { (primeira dose). }\end{array}$ \\
\hline Enalapril + Hidroclorotiazida & 14 & $\begin{array}{l}\text { Pode resultar em hipotensão postural } \\
\text { (primeira dose). }\end{array}$ \\
\hline Levotiroxina + Sinvastatina & 14 & Pode diminuir a eficácia de Levotiroxina. \\
\hline
\end{tabular}

Continua... 


\begin{tabular}{|c|c|c|}
\hline Interação medicamentosa & Prevalência & Mecanismo/Efeito \\
\hline $\begin{array}{c}\text { Ácido Acetilsalicílico + } \\
\text { Hidroclorotiazida }\end{array}$ & 10 & $\begin{array}{l}\text { Pode diminuir a eficácia do diurético e } \\
\text { anti-hipertensivo. }\end{array}$ \\
\hline $\begin{array}{l}\text { Ácido Acetilsalicílico }+ \\
\text { Succinato de Metoprolol }\end{array}$ & 9 & Pode diminuir o efeito anti-hipertensivo. \\
\hline Levotiroxina + Omeprazol & 9 & Pode aumentar os níveis de TSH. \\
\hline $\begin{array}{l}\text { Ácido Acetilsalicílico + } \\
\text { Captopril }\end{array}$ & 6 & Pode diminuir a eficácia do Captopril. \\
\hline $\begin{array}{l}\text { Ácido Acetilsalicílico + } \\
\text { Enalapril }\end{array}$ & 6 & Pode diminuir a eficácia do Enalapril. \\
\hline Amitriptilina + Diazepam & 6 & $\begin{array}{l}\text { Pode resultar em déficits psicomotores } \\
\text { (tempo de reação, diminuição, } \\
\text { decréscimo da vigilância). }\end{array}$ \\
\hline $\begin{array}{l}\text { Metformina + Succinato de } \\
\text { Metoprolol }\end{array}$ & 6 & $\begin{array}{c}\text { Pode resultar em hipoglicemia ou } \\
\text { hiperglicemia. }\end{array}$ \\
\hline Ibuprofeno + Hidroclorotiazida & 5 & $\begin{array}{l}\text { Pode diminuir a eficácia do diurético e } \\
\text { anti-hipertensivo. }\end{array}$ \\
\hline \multicolumn{3}{|c|}{ Interação Importante (Maior) $\quad n=45$} \\
\hline $\begin{array}{c}\text { Ácido Acetilsalicílico + } \\
\text { Fluoxetina }\end{array}$ & 7 & Risco aumentado de hemorragia. \\
\hline Anlodipino + Sinvastatina & 4 & $\begin{array}{l}\text { Pode resultar em aumento da exposição } \\
\text { sinvastatina e risco aumentado de } \\
\text { miopatia, incluindo rabdomiólise. }\end{array}$ \\
\hline Enalapril + Espironolactona & 3 & Pode resultar em hipercalemia. \\
\hline Fluoxetina + Propranolol & 3 & $\begin{array}{l}\text { Pode resultar em aumento do risco } \\
\text { de toxicidade propranolol, incluindo } \\
\text { bloqueio cardíaco completo. }\end{array}$ \\
\hline
\end{tabular}

Na Tabela 2 apresenta a relação dos fármacos $(n=10)$ que mais frequentemente apresentaram interações medicamentosas.

Tabela 2 - Fármacos mais envolvidos em interações medicamentosas potenciais

\begin{tabular}{c|c|c|c}
\hline Fármaco & $\begin{array}{c}\text { Número } \\
\text { de regimes } \\
\text { terapêuticos }\end{array}$ & $\begin{array}{c}\text { Prevalência de } \\
\text { interação nos regimes } \\
\text { terapêuticos }\end{array}$ & $\%$ \\
\hline Ácido Acetilsalicílico & 29 & 55 & 23,8 \\
\hline Hidroclorotiazida & 38 & 50 & 21,6 \\
\hline Enalapril & 25 & 30 & 13,0 \\
\hline Captopril & 22 & 26 & 11,2 \\
\hline Levotiroxina & 20 & 23 & 9,9 \\
\hline Sinvastatina & 23 & 25 & 10,8 \\
\hline Fluoxetina & 16 & 22 & 9,5 \\
\hline Omeprazol & 15 & 16 & 6,9 \\
\hline Diazepam & 14 & 19 & 8,2 \\
\hline Carbonato de Cálcio & 13 & 16 & 6,9 \\
\hline
\end{tabular}


A quantidade de interações medicamentosas encontradas por regime terapêutico independentemente do número de medicamentos prescritos variou de 01 a 10, 50,5\% e 1,8\% dos pacientes, respectivamente.

O manejo clínico sugerido pelas fontes terciárias ${ }^{16}$ para evitar ou lidar com as interações e seus percentuais de frequência estão descrito na Tabela 3.

Tabela 3 - Manejo clínico para as interações medicamentosas encontradas e sua frequência

\begin{tabular}{c|c}
\hline Manejo clínico & Frequência \\
\hline Monitorização do paciente & $170(73,6 \%)$ \\
\hline Ausência de precauções & $25(10,8 \%)$ \\
\hline Alteração de intervalo de administração & $21(9,1 \%)$ \\
\hline Ajuste de dose & $8(3,5 \%)$ \\
\hline Troca ou suspensão de um medicamento & $7(3,0 \%)$ \\
\hline
\end{tabular}

\section{Discussão}

A média de medicamentos $(3,9)$ utilizados por paciente neste estudo também está próxima da média encontrada $(2,8)$ no estudo em Vitória da Conquista/BA ${ }^{17}$ em prescrições da atenção primária, e em estudo realizado com usuários de antidepressivos que retiravam seus medicamentos na Unidade Básica de Saúde do município de São José do Inhacorál $\mathrm{RS}^{18}$, onde a média de medicamentos foi 4,03. 0 risco da ocorrência de interações medicamentosas aumenta com 0 aumento do número de medicamentos prescritos ${ }^{19}$. Em relação aos medicamentos mais utilizados pelos idosos, $42,9 \%$ estão relacionados aos problemas do sistema cardiovascular, sendo estes dados similares aos encontrados em outros estudos com idosos, 48,1\% no sul de Santa Catarina ${ }^{20}$ e 38,4\% em Hospital Universitário do Rio Grande do Sul21. Os medicamentos cardiovasculares têm sido amplamente utilizados pelos idosos já que as doenças cardiovasculares vêm liderando as causas de morbimortalidade nesses indivíduos ${ }^{22}$.

Quanto ao número de medicamentos utilizados pelos idosos, $32,3 \%$ deles faziam uso de cinco ou mais medicamentos, resultado inferior aos achados em Hospital Universitário do Rio Grande do Sul ${ }^{21}$ e em estudo realizado com idosos institucionalizados em uma cidade de Minas Gerais ${ }^{23}$, onde a prevalência de polifarmácia em idosos foi de 48,6\% e 67,2\%, respectivamente. A maior prevalência de enfermidades crônicas degenerativas nos idosos resulta na prática da polifarmácia, que impacta na segurança e qualidade de vida dessas pessoas, desencadeando reações adversas a medicamentos $\mathrm{e}$ ocasionando impactos na capacidade funcional do idoso em decorrência de interações medicamentosas ${ }^{24,25}$.

No estudo foram encontrados até 11 medicamentos por prescrição médica. Esse resultado é menor do que 0 encontrado no inquérito realizado em 577 idosos na cidade do Rio de Janeiro/RJ ${ }^{26}$ onde o número de medicamentos variou de 0 a 24, e aproxima-se do estudo realizado na cidade de Porto Alegre/RS com idosos residentes em instituição de longa permanência, onde o número de medicamentos utilizados foi de 0 a $13^{27}$. Os principais objetivos da associação medicamentosa são os de potencializar os efeitos terapêuticos, diminuir efeitos colaterais, diminuir doses terapêuticas, prevenir resistência, obter ações múltiplas e amplas, e proporcionar maior comodidade para o paciente. Entretanto, a maioria das associações ocorre inadvertidamente, em situação de politerapia, sendo danosa ao organismo ${ }^{28}$.

Muitos estudos realizados sobre interações medicamentosas são referentes a determinadas classes terapêuticas e geralmente realizados em pacientes hospitalizados, no entanto, encontram resultados semelhantes aos estudos realizados na atenção primária, com grande número de tratamentos medicamentosos apresentando interações entre os medicamentos. Cita-se o estudo realizado em pacientes internados em hospital do sudoeste da Bahia ${ }^{29}$ onde $46,5 \%$ das prescrições analisadas apresentaram pelo menos uma interação medicamentosa potencial, comparado a um estudo já citado anteriormente, realizado em Vitória da Conquista, do mesmo Estado, que encontrou frequência de interações medicamentosas nas prescrições de 48,9\%, com média de 0,80 interações por prescrição ${ }^{17}$, aproximando-se do encontrado neste estudo que foi de $54,4 \%$ das prescrições médicas contendo pelo menos uma interação medicamentosa potencial. Neste estudo o número de medicamentos prescritos impactou na quantidade de interações, corroborando com o encontrado no estudo realizado em idosos hipertensos, na cidade de Ribeirão Preto/SP, onde o número de interações medicamentosas foi diretamente proporcional ao número de medicamentos ${ }^{30}$. 
As interações medicamentosas potenciais podem ser classificadas de acordo coma gravidade como: contraindicada, quando os medicamentos são contraindicados para uso concomitante; importante ou maior, quando a interação pode representar perigo à vida e/ou requerer intervenção médica para diminuir ou evitar efeitos adversos graves; moderada, quando a interação pode resultar em exacerbação do problema de saúde do paciente e/ou requerer uma alteração no tratamento; secundária ou menor, quando a interação resultaria em efeitos clínicos limitados. As manifestações podem incluir um aumento na frequência ou gravidade dos efeitos colaterais, mas geralmente não requerem uma alteração importante no tratamento; e desconhecida, quando o efeito é desconhecido ${ }^{16}$.

As interações medicamentosas em potencial com gravidade moderada apresentaram maior prevalência, contrariando estudo realizado com usuários de antidepressivos que retiravam seus medicamentos em Unidade Básica de Saúde no interior do Rio Grande do Sul, no qual prevaleceram as interações com gravidade maior ${ }^{18}$. Destaca-se a Hidroclorotiazida associada ao Enalapril que pode resultar em hipotensão postural ${ }^{16}$, definida como uma queda na pressão sistólica de ao menos $20 \mathrm{mmHg}$, ou por queda da pressão diastólica de $10 \mathrm{mmHg}$ após o indivíduo levantar-se ${ }^{31}$. Este efeito leva a uma maior fragilidade física nos idosos, podendo aumentar o perfil de mortalidade deste grupo etário ${ }^{3}$. Em estudo realizado na Unidade Básica de Saúde de Ribeirão Preto/SP com 40 idosos, os fármacos Hidroclorotiazida e o Ácido Acetilsalicílico foram os mais prevalentes e este último como o responsável pela maioria das interações ${ }^{30}$, corroborando com o encontrado nesse estudo. 0 ácido acetilsalicílico, tem sido utilizado há muito tempo em idosos, na prevenção de eventos trombolíticos ${ }^{32}$.

No estudo, dois idosos chegaram a apresentar até dez interações medicamentosas e cinquenta apresentaram pelo menos uma interação. As interações medicamentosas são consideradas erros de medicação, com risco de dano em função da sua ocorrência, tornando-se relevante identificar a natureza e determinantes destes erros, como forma de encaminhar ações preventivas ${ }^{33}$. Destaca-se a importância da adoção de procedimentos de manejo clínico a fim de evitar ou reduzir a intensidade das interações medicamentosas durante a condução do tratamento terapêutico. Na prática assistencial as interações medicamentosas são determinadas, muitas vezes, pela condição clínica dos pacientes ${ }^{34}$. No caso do idoso, pela complexidade da farmacoterapia e múltiplas patologias, é geralmente indicado o uso concomitante de fármacos, mas para garantir uma farmacoterapia segura e efetiva é importante que a equipe de saúde saiba identificar as interações e reconhecer as estratégias de monitorização $0^{35}$.

Na maioria dos países desenvolvidos a Atenção Farmacêutica já é realidade e tem demonstrado ser eficaz na redução de agravamento dos portadores de patologias crônicas e de custos para o sistema de saúde. No entanto, no Brasil esta prática ainda é incipiente e alguns fatores dificultam sua implantação. Apesar de haver estudos demonstrando que a intervenção farmacêutica por meio de ações educativas e de aconselhamento sobre regime terapêutico trazem benefícios à saúde do paciente e para o processo de promoção da saúde ${ }^{36,37}$, ainda sofremos de carência de documentação científica que possibilite demonstrar aos gestores dos sistemas de saúde que a inserção deste profissional representa investimento e não custo.

\section{Considerações Finais}

As interações medicamentosas podem ocasionar vários problemas à farmacoterapia do idoso como reduzir absorção, concentrações e eficácia, causar toxicidade e risco aumentado de hemorragias, resultar em efeitos maiores e prolongados, resultar em hipotensão postural, interferirem em exames laboratoriais, resultar em déficits psicomotores, hipoglicemia, hiperglicemia, miopatia, rabdomiólise, hipercalemia, entre outros, comprometendo o tratamento e a segurança do idoso, interferindo na sua funcionalidade e na sua qualidade de vida. Mesmo que nem todas as interações medicamentosas possam ser prevenidas, a propagação do conhecimento entre os profissionais de saúde, constitui um dos principais instrumentos de prevenção, visando sempre a otimização dos regimes terapêuticos e o uso racional de medicamentos. Desta forma, é de suma importância o conhecimento das influências que um fármaco pode exercer sobre o outro quando utilizados concomitantemente, sendo a administração correta dos medicamentos um fator determinante para minimizar ou evitar a interação medicamentosa.

Desta maneira, este estudo, tornou-se uma ferramenta importante para o planejamento de ações na farmácia municipal em prol da melhor qualidade de vida desses idosos. Entre as ações desenvolvidas destacam-se, a capacitação da equipe responsável pela dispensação dos medicamentos e a orientação farmacêutica realizada aos idosos, visando identificação de potenciais interações medicamentosas. Uma limitação deste estudo, foi que o local onde foi desenvolvida a pesquisa, ocorre apenas a dispensação de medicamentos e não a prescrição dos mesmos, dificultando o acesso aos prescritores. 


\section{Referências}

1. BRASIL. Ministério da Saúde. Secretaria de Atenção à Saúde. Departamento de Atenção Básica. Envelhecimento e saúde da pessoa idosa. Brasília: Ministério da Saúde, 2011.

2. IBGE. Instituto Brasileiro de Geografia e Estatística. [Internet]. 2010. [acesso em 2016 fevereiro 04]. Disponível em: http://censo2010.ibge.gov.br/resultados.html.

3. Secoli SR. Polifarmácia: interações e reações adversas no uso de medicamentos por idosos. Rev Bras Enfer, Brasília. 2010;63(1):136-40.

4. Corrêa LM, Rodrigues $C$, Macedo LC. Avaliação farmacoterápica em pacientes de uma instituição geriátrica da região centro - ocidental do Paraná, Brasil. SaBios: Rev. Saúde e Biol.2016; 11(1):22-30.

5. Carvalho MFC, Romano-Liber NS, Bergsten-Mendes G, Secoli SR, Ribeiro E, Lebrão ML, Duarte YAO. Polifarmácia entre idosos do Município de São Paulo - Estudo SABE. Rev Bras Epidemiol. 2012;15(4):817-27. Disponível em: http:// www.scielo.br/scielo.php?script=sci_arttext\&pid=S1415790X2012000400013 doi: 10.1590/S1415-79X2012000400013.

6. Ribeiro $\mathrm{AQ}$, Rozenfeld $\mathrm{S}$, Klein $\mathrm{CH}$, César CC, Acurcio FS. Inquérito sobre uso de medicamentos por idosos aposentados, Belo Horizonte, MG. Rev Saúde Pública 2008;42:724-32. Disponível em: http://www.scielo.br/scielo. php?script=sci_arttext\&pid=S003489102008000400020\&lng=en. doi:10.1590/S0034-89102008005000031.

7. Mibielli P, Rozenfeld S, Matos GC, Acurcio FA. Interações medicamentosas potenciais entre idosos em uso dos antihipertensivos da Relação Nacional de Medicamentos Essenciais do Ministério da Saúde do Brasil. Cad.Saúde Pública, Rio de Janeiro. 2014;30(9):1947-56. Disponível em: http://www.scielo.br/pdf/csp/v30n9/0102-311X-csp-30-9-1947.pdf. doi: 10.1590/0102-311X00126213.

8. Moura C, Acurcio F, Belo N. Drug-drug interactions associated with length of stay and cost of hospitalization. J Pharm Pharmaceut Sci 2009;12(3):266-72.

9. Bisson MP. Farmácia clínica \& atenção farmacêutica. 2. ed. Rev. e atual. São Paulo: Manole. 2007;p.371.

10. Araújo RC. Interações Medicamentosas no Idoso. In: Silva P. Farmacologia. $6^{a}$ ed. Rio de Janeiro: Guanabara Koogan. 2002;p. 162-5.

11. Monteiro SCM, Belfort IKP, Sousal WR, Barros CS, Campos KVS. Estudo de potenciais interações medicamentosas em pacientes hipertensos. Infarma Ciências Farmacêuticas. 2015;27(2):117-25. Dsiponível em: http://revistas.cff.org. br/?journal=infarma\&page=article\&op=view\&path[]=723. doi: 10.14450/2318-9312.

12. Kawano DF, PereirA LRL, Ueta JM, Freitas O. Acidentes com os medicamentos: como minimizá-los? Rev Ciênc Farmacêut. 2006;42(4):487-95. Dsiponivel em: http://www.scielo.br/pdf/rbcf/v42n4/a03v42n4.pdf. doi: 10.1590/ S151693322006000400003.

13. Beyth RJ \& Shorr RI. Uso de medicamentos. In Duthie EH \& Katz PR. Geriatria prática (3a ed.).2002;Ed. Revinter, Rio de Janeiro.

14. Varallo FR, Costa MA, Mastroianni PC. Potenciais interações medicamentosas responsáveis por interações hospitalares. Rev Ciênc Farm Básica Apl. 2013; 34(1):79-85.

15. Bechi VS. Atenção Farmacêutica: uso racional de medicamento na rede pública pelos idosos. Facider Revista Científica, Colider. 2015; $\left(n^{0} 7\right)$.

16. MICROMEDEX® Solutions. [Internet]. Acessado: 2016 Janeiro 20. Disponível em: http://www-micromedexsolutions com.ez127.periodicos.capes.gov.br/micromedex2/librarian.

17. Leão DFL, Moura CS, Medeiros DS. Avaliação de interações medicamentosas potenciais em prescrições da atenção primária de Vitória da Conquista (BA), Brasil. Ciência\&Saúde Coletiva. 2014;19(1):311-18. Disponível em: http://www. scielosp.org/pdf/csc/v19n1/1413-8123-csc-19-01-00311.pdf. doi: 10.1590/1413-81232014191.2124.

18. Schenkel M, Simão J, Schwanbach KH, Colet CF. Interação medicamentosa em usuários de antidepressivos do sistema público de um município do sul do Brasil. Ciência\&Saúde.2015;8(3): 107-14.

19. Marquito AB, Fernandes NMS, Colugnati FAB, Paula RB. Interações medicamentosas potenciais em pacientes com doença renal crônica. J Bras Nefrol. 2014;36(1):26-34. Dsiponível em: http://www.scielo.br/pdf/jbn/v36n1/0101-2800jbn-36-01-0026.pdf. doi: 10/5935/0101-2800.20140006.

20. Bagatini F, Blatt CR, Maliska G, Trespash GV, Pereira IA, ZimemermannAF, etal. Potenciais interações medicamentosas em pacientes com artrite reumatoide. Rev Bras Pneumatol 2011;51(1): 20-39. Disponível em: http://www.scielo.br/scielo. php?script=sci_arttext\&pid=S048250042011000100003. doi: 10.1590/S0482-50042011000100003. 
21. Gauterio DP, Santos SSC, Strapasson CMS, Vidal DAS, Piexak DR. Uso de medicamentos por pessoas idosas na comunidade: proposta de ação de enfermagem. Rev Bras Enferm. 2013;66(5):702-8. Disponível em: http://www.redalyc. org/pdf/2670/267028883010.pdf. doi: 10.1590/S0034-71672013000500010.

22. Ferreira CCC, Peixoto MRG, Barbosa MA, Silveira EA. Prevalência de fatores de risco cardiovascular em idosos usuários do Sistema Único de Saúde de Goiânia. Arq Bras Cardiol. 2010;95(5): 621-28. Disponível em: http://www.scielo. br/scielo.php?script=sci_arttext\&pid=S0066-782X2010001500010. doi: 10/1590/S0066-782X2010005000141.

23. Fochat RC, Horsth RBO, Sette MS, Raposo NRB, Chicourel EL. Perfil de utilização de medicamentos por idosos frágeis institucionalizados na Zona da Mata Mineira, Brasil. Rev Ciênc Farm Básica Apl. 2012; 33(3):447-54.

24. Varallo FR, Ambiel ISS, Nanci LO, Galduróz JCF, Mastroianni PC. Assessment of pharmacotherapeutic safety of medical prescriptions for elderly residents in a long-term care facility. Braz J Pharm Sci 2012;48(3):477-85. Disponível em: http://www. scielo.br/scielo.php?script=sci_arttext\&pid=S1984-82502012000300015. doi: 10.1590/S1984-82502012000300015.

25. Oliveira MG, Amorim WW, Rodrigues VA, Passos LC. Access of Brazilian elders to potentially inappropriate medications. Rev APS. 2011;14(3):258-65.

26. Mibielli P, Rozenfeld S, Matos GC, Acurcio FA. Interações medicamentosas potenciais entre idosos em uso de antihipertensivos da Relação Nacional de Medicamentos Essenciais do Ministério da Saúde do Brasil. Cad Saúde Pública, Rio de Janeiro. 2014;30(9):1947-56. Disponível em: http://www.scielo.br/pdf/csp/v30n9/0102-311X-csp-30-9-1947.pdf. doi: 10.1590/0102-311X00126213.

27. Gerlack LF, Cuentro VS, Estrela MFB, Karnikowski MGO, Pinho DLM, Bós AJG. Interações medicamentosas na farmacoterapia prescrita a idosos residentes em uma instituição de longa permanência brasileira. Estud. Interdiscipl. Envelhec., Porto Alegre. 2014;19(2):439-52.

28. Osório de Castro CGS. Interações medicamentosas. In: Fuchs FD, Wannmacher L. Farmacologia clínica: fundamentos da terapêutica racional. $4^{\mathrm{a}}$ ed. Rio de Janeiro: Guanabara Koognan;2010.

29. Piedade DV, Silva LAF, Lemos GS, Júnior GLV, Lemos LB. Interações medicamentosas potenciais em prescrições, contendo antimicrobianos de uso restrito, de pacientes internados em um hospital no interior da Bahia. Medicina (Ribeirão Preto) 2015;48(3):295-307.

30. Pinto NBF, Vieira LB, Pereira FMV, Reis AMM, Cassiani SHB. Interações medicamentosas em prescrições de idosos hipertensos: prevalência e significância clínica. Rev enferm UERJ. Rio Janeiro. 2014;22(6):785-91.

31. Metzler M, Duerr S, Granata R, Krismer F, Robertson D, Wenning GK. Neurogenic orthostatic hypotension: pathophysiology, evaluation, and management. J Neurol. 2013;260:2212-9.

32. Ansara Aj, Nilsy AS, Arif AS, Koehler JM, Nordmeyer ST. Aspirin dosing for the prevention and treatment of ischemic stroke: an indication-specific review of the literature. Ann Pharmacother. 2010;44: 851-62. Disponível em: http:// digitalcommons.butler.edu/cgi/viewcontent.cgi?article=1040\&context=cophs_papers. doi: 10.1345/aph.1M346.

33. BRASIL. Ministério da Saúde. Secretaria de Ciência, tecnologia e Insumos. Departamento de Assistência Farmacêutica e Insumos estratégicos. Formulário terapêutico nacional 2010: Rename 2010.2 ed. Brasília: Ministério da Saúde, 2011. 34. Reis AM, Cassiani SH. Evaluation of three brands of drug interaction software for use in intensive care units. Pharm World Sci. 2010;32: 822-8.

35. Pinto NBF, Vieira LB, Pereira FMV, Reis AMM, Cassiani SHB. Interações medicamentosas em prescrições de idosos hipertensos: prevalência e significância clínica. Rev Enferm UERJ, Rio de Janeiro. 2014;22(6):735-41. Disponível em: http://www.facenf.uerj.br/v22n6/v22n6a02.pdf. doi: http://dx.doi.org/10.12957/reuerj.2014.7111.

36. Partata AK, Alves LK, Alves AJ. Atuação do farmacêutico na promoção e restauração da saúde de pacientes idosos que fazem uso de polimedicação. Rev ITPAC. 2010;3(2):5-15.

37. Costa EM, Rabelo ARM, Lima JG. O farmacêutico na promoção da saúde e prevenção de agravos. Rev Ciênc Farm Básica Apl. 2014;35(1):81-8. 


\section{Cristiane Carla Dressler Garske}

Endereço para correspondência - Rua: Manaus, $n^{\circ} 685$, Ap 219, Bloco E, Bairro: Vila Schulz, CEP: 96845500 , Santa Cruz do Sul, RS, Brasil.

E-mail: crisdressler2005@yahoo.com.br Lattes: http://lattes.cnpq.br/7469653524983533

Mariana Portela de Assis - portela1311@yahoo.com.br Ana Paula Helfer Schneider - anahelfer@unisc.br Ediberto de Oliveira Machado - emachado@unisc.br Lisoni Muller Morsch - Imorsch@unisc.br

\section{Enviado em 09 de abril de 2016.} Aceito em 26 de julho de 2016. 